\title{
EL PERFIL DEL CONTADOR PÚBLICO Y DE LOS ESTUDIOS DE POSTGR'ADO
}

Dr. ERNESTO A. POLAR FALCÓN (*)

La Facultad de Ciencias Contables de la UNMSM, desde su fundación hasta la fecha, siempre ha tenido muy claro el perfil del Contador Público que forma profesionalmente. Su filosofia educativa está cimentada en cumplir con dos objetivos: el primero, mantener el alto nivel de formación del Contador Público que se ofrece año tras año a la sociedad peruana; y el segundo, destacar su liderazgo de educación universitaria en el consenso de dirección y ejemplo a nivel de las Facultades de la Universidad Peruana, que actualmente ofrecen la carrera de Contador Público.

Prueba de ello, son las legiones de Contadores Públicos Sanmarquinos que hoy en día, trabajan en el sector público y privado, en altos cargos de gran responsabilidad; en el desarrollo de la actividad independiente profesional brindando principalmente los servicios de contabilidad, auditoría y consultoría. También dirigiendo los destinos de la profesión de Contador Dúblico en las instituciones tutelares de la profesión. En cuanto a dirección y ejemplo a nivel de la Universidad Peruana, ¿qué Facultad que forma Contadores Públicos en el Perú no ha tomado como modelo el Currículo de Estudios de la Facultad de Ciencias Contables de la UNMSM?
Al respecto, el Contador Público Dr. Antonio C. Nasi, Presidente de la Asociación Interamericana de Contabilidad, vigente hasta Noviembre del 2001, mes en que se cumplirán sus dos años de labor de servicios a toda América en este importante cargo, dice enfáticamente que ninguna Universidad del mundo puede mantener vigente más de cinco años, el mismo Perfil de Contador Público que aplica en su formación profesional.

En esto, tiene razón, por cuanto frente a los grandes y rápidos cambios que experimenta este mundo globalizado, se tiene que ir modificando el Perfil de Contador Público, para que vaya a la par de los vertiginosos giros que se dan en los negocios y en las finanzas nacionales e internacionales. $\mathrm{El}$ Dr. Nasi está pues empeñado en incentivar a las autoridades universitarias y profesionales de América, a elevar constantemente la calidad profesional del Contador Público.

Pero, antes de presentar el Perfil del Contador Público que tiene la Facultad de Ciencias Contables de la UNMSM, es menester conocer un poco de las bases que forman un Perfil Profesional. Para esto, a continuación se presentan resumidamente los siguientes conceptos:

\footnotetext{
- ) Docente Principal de la Facultad de Ciencias Contables de la UNMSM.
} 


\section{A. CONCEPTOS BÁSICOS}

\section{Educación}

Educacion es una praxis socio histórica, cultural, que en armonía con la naturaleza busca el desarrollo de las potencialidades del ser humano, como valores, conocimientos, actitudes, significados y capacidad de autodeterminación. Su finalidad implícita es hacer al hombre más humano, plasmar su humanidad en relaciones que se transmiten generacionalmente.

En este sentido la educación universitaria tiene como finalidad fundamental 'No solamente la comunicación de un saber adquirido sino el despertar la iniciativa, la creatividad, el espíritu de búsqueda de cuanto intervienen en ella". La formación académica debe reflejar el ser de la Universidad, su ligación profunda con el saber, su intento de universalidad y su relación con la sociedad.

\section{Currículo}

Es una acción humana educativa, prevista real integrada y sistemática, que al plasmarse en un proyecto prescribe con fle xibilidad principios y orientaciones, reconstruye reconocimientos y experiencias en todas sus dimensiones y variables.

Su finalidad específica es viabilizar la formación del potencial humano a través del proceso de enseñanza-aprendizaje, en permanente interacción con su entorno sociocultural.

\section{Planificación Curricular}

Proceso inmerso en la planificación educativa y por ende en la planificación general del país. Se diseña en base a dos marcos fundamentales, un marco referencial y un marco conceptual. Ambos sintetizan su in- fluencia en el eje central del currículum llamado PERFIL PROFESIONAL.

La planificación curricular prevé de manera estructurada e inteligente las diversas etapas del trabajo educativo, con la finalidad de alcanzar en el estudiante los objetivos propuestos. En función de estos objetivos se diseña la estructura curricular, la misma que al articular elementos y sujetos del currículo, orienta coherentemente el proceso enseñanza aprendizaje, facilitando así, la formación de un egresado de calidad socio personal.

En la planificación curricular interactúan tres componentes indispensables, los sujetos: educando, educador y comunidad educacional; los procesos curriculares: investigación, programación, organización o administración, implementación, ejecución y evaluación curricular; y los elementos: objetivos, contenidos, estrategias metodológicas, medios y materiales educativos, infraestructura y tiempo.

\section{Programación Curricular}

Conjunto de acciones educativas sucesivas previstas en el tiempo en forma organizada y concreta. Proceso orientado que configura anticipadamente la interacción de los sujetos y elementos del currículo con la finalidad de suscitar experiencias motivadoras de enseñanza aprendizaje congruentes con los objetivos y el perfil profesional.

\section{B. PROPUESTA DE DISEÑO CURRICULAR}

Concretar el diseño curricular en su fase inicial implica empezar por distinguir los marcos fundamentales que esclarecen su elaboración. Se indica así al marco Referencial y al Marco Conceptual los que, 
a manera de vertientes sustantivas, interactúan y alimentan progresiva y permanentemente al proceso curricular.

\section{Marco Referencial}

Llamado también diagnóstico, viene a constituir el estudio minucioso y objetivo de los problemas más serios y frecuentes que ostenta una realidad.

Su utilidad reside en permitir una aproximación y conocimiento de la situación estructural en la cual se hallan el contexto general, su entorno educativo, el potencial humano concurrente a una formación profesional, la institución formadora y los currículos; así como, los recursos necesarios para la formación universitaria.

\section{Marco Conceptual}

Con la finalidad de visualizar la problemática a estudiar y encontrar las prioridades más significativas, se debe recurrir a los fundamentos básicos del currículo como son: los filosóficos doctrinarios, los cientificos tecnológicos, los andragógicos, legales, políticas, socioeconómico y otros que se encuentran en el Marco Conceptual. El siguiente cuadro es expresión de este concepto:

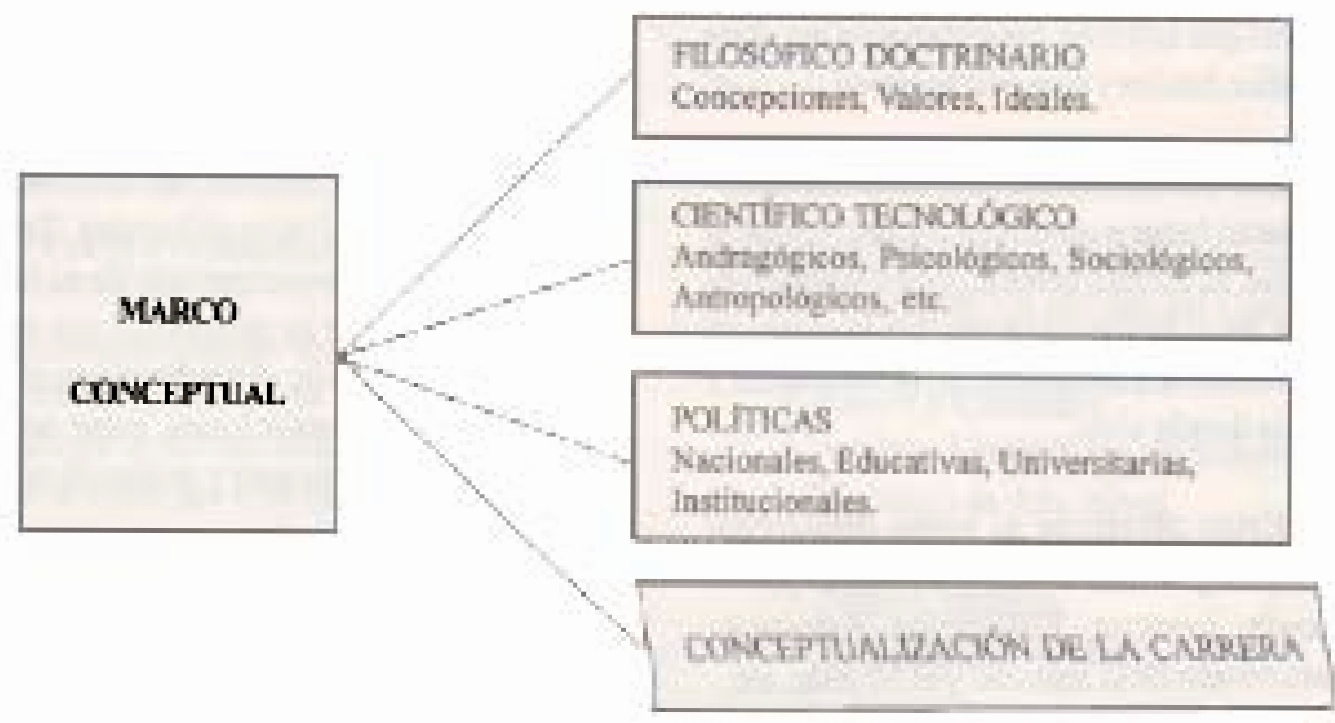

Asimismo, es importante comprender el siguiente proceso de perfil educacional:

\section{«... PERFIL EDUCACIONAL}

Asumiendo la importancia de la formación general como base de una educación integral ulterior del egresado, sin distinción de carrera o especialidad, se procede a diseñar el perfil educacional.

Formular este perfil implica postular a dos diseños de perfil. Uno correspondiente a su Perfil Básico, y otro, a la formación en su carrera denominado Perfil Profesional.

\section{a. Perfil Básico}

Lograr el Perfil Básico requiere optar por una perspectiva bidimensional, una personal y otra social. 
La primera dimensión es para considerar los rasgos característicos, cualidades o atributos de la persona. El cómo debe SER como ser humano, su realización como tal, sus actitudes, sus valores; así como también, lo que debe SABER como agente cultural. Su desarrollo cognoscitivo científico básico, todo conocimiento fundamental de la generalidad de las ciencias sin distinción de carrera profesional.

La segunda dimensión está dada en el aspecto SOCIAL, lo que debe HACER, sus destrezas, modelos de conducta, sus interacciones y sus habilidades.

Estas dos dimensiones deben ser interrelacionadas con las áreas de la formación general que son tres: área humanística, área científico básica y área institucional.

Las características del área humanística darán lugar a objetivos formativos de la persona, su cultura general, una visión actualizada de su ubicación como ser humano en su contexto sociocultural, en la naturaleza y en país donde vive.

Estos objetivos se verán desagregados en asignaturas como: Filosofia, Antropología, Lengua, Educación Artística, Cosmovisión de la realidad y el Mundo, etc.

Los rasgos que tipifican la formación científico básica obedecerán a objetivos de entorno a las ciencias formales y factuales más relevantes como: ser consciente de SL ser biopsíquico, reflexivo, agudo, analítico. observador y otros. Las asignaturas orientadas a estos propósitos serán: matemáticas. biología, sociología, psicología, lógica, etc.

En lo que a formación institucional se refiere, todo egresado exhibe un sello peculiar de la institución que lo formó.

Su investidura, alma mater, su sentido de pertenencia, la identificación con sus principios, éxitos o carencias, el estar comprometidos con sus símbolos hasta defenderlos y llevar en alto el nombre de la institución en los diversos actos de su desempeño.

Estas características se alcanzan a través de las actividades curriculares, el modelo de sus maestros, las acciones académicas, recreativas o culturales. También contribuyen el conocimiento de su historia institucional, de su patrón, de sus más logrados exponentes; el predicar con el ejemplo, con sus orientaciones, estar en coherencia con sus normas y gozar de sus estímulos y ayudas.

Visualizadas estas cualidades se procede a sistematizarlas y sintetizarlas a fin de ver concretado el diseño del PERFIL BÁSICO: 


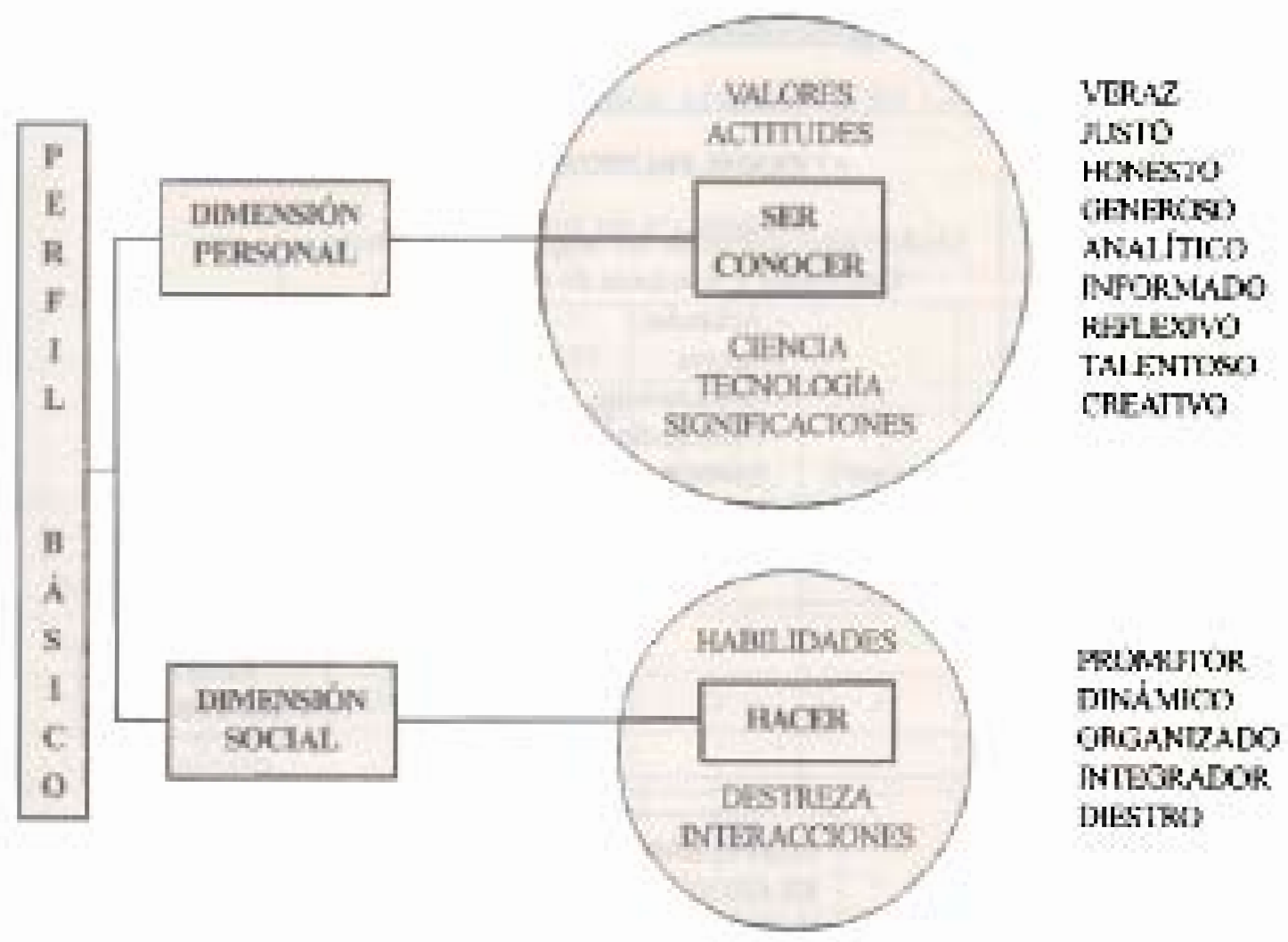

\section{b. Perfil Profesional}

En base al trabajo anterior es hora de preguntarse: ¿Cuál es la imagen de profesional que la universidad quiere formar? ¿Cómo se describe o se concibe la carrera profesional que se debe impartir? ¿Cómo es la Ingeniería, las Ciencias de Comunicación, las Ciencias Contables, la Medicina, etc?

Absueltas estas interrogantes se debe considerar tres aspectos:

b.1. La descripción de la carrera.

b.2. Un análisis de las funciones y tareas que se deben cumplir.

b.3. Los campos de influencia o de ejercicio profesional.

En una matriz de doble entrada se ubicará en la línea horizontal las áreas de formación profesional: Científico Básica Especializada y de Práctica Pre-Profesional y en la línea vertical, los campos de acción del futuro egresado; según sea el caso que se trate. Ejemplo: En el caso del Contador Público:

- Asesor y Consultor.

- Analista Financieroy de Sistemas.

- Ejecutivo de Alto Nivel.

- Auditor.

- Perito Contable.

- Docente Universitario.

- Contralor.

- Investigador.

- Administrador de la Información Computarizada Contable.

- Contador de Costos.

En la Intersección de ambas variables se ubicarán las funciones que debe cumplir, desagregándolas en sus tareas más importantes. Al igual que en el caso anterior, serán sistematizadas y sintetizadas en áreas o funciones a fin de que al integrarlas permitan obtener el PERFIL PROFESIONAL. 
A continuación se sintetiza gráficamente el Perfil Profesional:

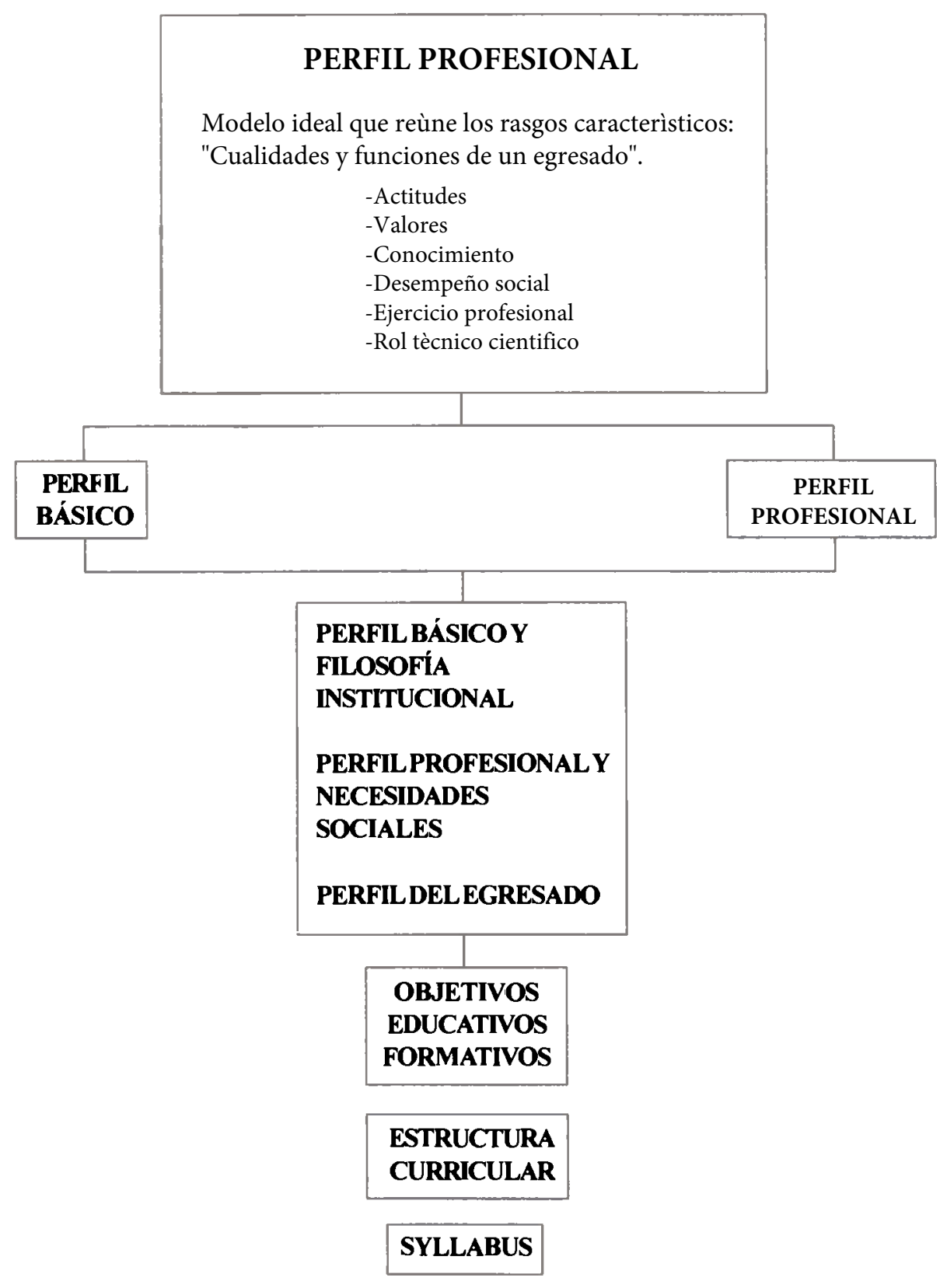

Los tres Currículos o Planes de Estudios de la década de los noventa, que ha aplicado la Facultad de Ciencias Contables de la UNMSM, en la formación de nuevos Contadores Públicos, es una prueba de los rápidos cambios en la profesión y del interés académico de la Facultad de mantenerse acorde con dichos cambios.
A continuación, se muestra un cuadro conteniendo los tres Planes de Estudio que aplicó la Facultad en la década del noventa, distribuidos en dieciséis áreas de Estudios: 
FACULTAD DE CIENCIAS CONTABLES

PLANES DE ESTUDIOS APLICADOS EN LA

DÉCADA DE LOS NOVENTA

AÑOS DE CAMBIOS

\begin{tabular}{|c|c|c|c|c|c|c|}
\hline \multirow[t]{2}{*}{$\mathbf{N}^{\mathbf{0}}$} & \multirow{2}{*}{$\begin{array}{l}\text { Área de } \\
\text { Estudio }\end{array}$} & \multirow{2}{*}{$\begin{array}{c}1992 \\
\text { Cursos } \\
\text { Semest. }\end{array}$} & \multicolumn{2}{|c|}{1995} & \multirow{2}{*}{$\begin{array}{c}1996 \\
\text { Cursos } \\
\text { Anuales }\end{array}$} & \multirow[b]{2}{*}{ Porcentajes } \\
\hline & & & $\begin{array}{l}\text { Cursos } \\
\text { Anuales }\end{array}$ & $\begin{array}{l}\text { Cursos } \\
\text { Semest. }\end{array}$ & & \\
\hline 1 & Contabilidad & 17 & 5 & 5 & 5 & 17 \\
\hline 2 & Matemáticas & 8 & 5 & - & 4 & 14 \\
\hline 3 & Introducción a la Profesión & - & - & - & - & - \\
\hline 4 & Cosmovisión Sociológica & 6 & 2 & 4 & 4 & 14 \\
\hline 5 & Comunicaciones & - & - & - & 1 & 3 \\
\hline 6 & Administración & 4 & 2 & 1 & 1 & 3 \\
\hline 7 & Economía & 5 & 1 & 1 & 2 & 7 \\
\hline 8 & Derecho Comercial & 3 & 2 & - & 1 & 3 \\
\hline 9 & Derecho Laboral & 1 & - & 1 & 1 & 3 \\
\hline 10 & Costos & 2 & 2 & - & 1 & 3 \\
\hline 11 & Informática y Computadores & 1 & 2 & - & 1 & 3 \\
\hline 12 & Finanzas & 2 & 2 & 2 & 1 & 3 \\
\hline 13 & Derecho Tributario & 4 & 1 & 1 & 2 & 7 \\
\hline 14 & Auditoría & 7 & 1 & 5 & 4 & 14 \\
\hline 15 & Ética & - & - & - & 1 & 3 \\
\hline 16 & Metodología de la Investigación & $\cdot$ & - & 1 & 1 & 3 \\
\hline & TOTAL DE CURSOS & 60 & 25 & 21 & 30 & 100 \\
\hline
\end{tabular}

Si se analizan estos tres Planes de Estudios, se pueden presentar los siguientes comentarios:

1. En 1992 se estudiaba la carrera de Contador Público con el marco de sesenta cursos semestrales. En 1995 se tenía un Plan de Estudios con veinticinco cursos anuales y veintiún cursos semestrales. Y en 1996 se aplica el vigente Plan de Estudios con treinta cursos anuales.
2. El vigente Plan de Estudios 1996, ya tiene la primera Promoción 2000, como resultado de aplicar un nuevo esquema curricular, en el cual el $17 \%$ son cursos de contabilidad, $14 \%$ son cursos de matemáticas, el $14 \%$ son cursos de humanidades; el $14 \%$ son cursos de auditoría; el $7 \%$ son cursos de economía; y el $7 \%$ son cursos de derecho tributario. Estas seis áreas totalizan $73 \%$ y el resto de áreas de curso totalizan el $27 \%$. Al finalizar el presente año, 
saldrá la Segunda Promoción 2001, también con este nuevo esquema. ¿Tenemos que cambiar el vigente Plan de Estudios? Es indudable, por cuanto la profesión del Contador Público a nivel mundial, ya tiene singulares cambios que deben ser estudiados para implementarlos en nuevo Plan de Estudios.

3. ¿Se podrá estructurar un nuevo Plan de Estudios en los tres últimos meses del 2001 e iniciar uno nuevo en el 2002? ¿Se tendrá el personal docente debidamente entrenado para el dictado de nuevos cursos en estos tres meses del 2001 ? Estos son candentes temas que deberán ser estudiados con mucho cuidado, por la gran responsabilidad que se tiene en cambiar el Plan de Estudios, para lograr el Contador Público que necesita el Perú en los dos primeros quinquenios del presente siglo XXI.

El Perfil del Contador Público que tiene como objetivo el Plan de Estudios de 1996 de la Facultad de Ciencias Contables de la UNMSM es el siguiente:

\section{PERFIL PROFESIONAL DEL CONTADOR PÚBLICO}

En un contexto mundial de globalización de la economía y de los sistemas de información se requiere de un profesional Contador Público capaz de evaluar los hechos económicos y financieros así como diseñar, administrar y dictaminar sistemas de información financiera que posibiliten la toma de decisiones, orientado a un enfoque global y estratégico en un medio de alta competencia.

El profesional Contador Público debe tener las siguientes cualidades

\section{Personales:}

El profesional Contador Público, debe ser una persona con valores éticos, respete la dignidad de la persona; sepa valorar el medio ambiente, y tener una alta vocación de servicio a la comunidad. Debe tener espíritu emprendedor, asumir liderazgo y la cultura de la calidad.

Debe contar con habilidad para la comunicación oral y escrita. Debe ser persona con afán de constante superación y digno miembro de una comunidad.

Debe ser creativo, ordenado, disciplinado proactivo, puntual, responsable y tener sentido de organización y responsabilidad.

1.1 Intelectuales:

El Contador Público debe poseer razonamiento lógico, inductivo, deductivo, habilidad matemática, lectura crítica y debe ser metódico.

\subsection{Sociales:}

El Profesional Contador Público debe ser capaz de interrelacionarse con el medio social, con vocación de servicio y sentido social. Estará capacitado para asumir posiciones de liderazgo en la comunidad.

\section{De Formación Profesional:}

El Contador Público debe tener una formación profesional de nivel superior que comprenda los siguientes aspectos:

\subsection{Humanista}

Comprende una formación ética, filosófica, sociológica, y de análisis e interpretación de la realidad nacional, en forma integral que contribuya al liderazgo. El Proceso de globalización de la economía 
exige que el profesional domine un idioma extranjero, preferentemente el Inglés.

\subsection{Científica y Tecnológica}

El profesional debe contar con una sólida base de doctrina contable; conformada por la teoría, principios, normas, métodos y prácticas contables. El proceso de aprensión debe ser ordenado y secuencial tratando de relacionar la teoría con la práctica.

La formación profesional debe considerar la Contabilidad Gerencial orientada a la toma de decisiones estratégicas; las Finanzas para evaluar la estructura financiera de la empresa, la rentabilidad y riesgos de inversión. La Contabilidad Financiera desde el registro contable por medios computarizados, la formulación de los Estados Financieros Consolidados y convertidos a moneda extranjera. La auditoría en sus especialidades de sistemas computarizados, financiera, operativa, tributaria y del medio ambiente. La tributación para el estudio, análisis e interpretación de los dispositivos de carácter tributario; comparado con legislación tributaria de los países vecinos. Las matemáticas y la estadística proporcionan los fundamentos para el análisis matemático y financiero. Los fundamentos para la gestión y toma de decisiones en la empresa son proporcionados por las asignaturas de administración; en tanto que el comportamiento de variables micro y macro económicos que afectan en los hechos económicos se obtienen de las asignaturas de Economía.

En el campo del Derecho, además de la parte elemental; se aplica el Derecho Financiero.

\subsection{Investigación}

La investigación será parte integrante de la formación profesional a través de los cursos que conforman el Currículo de
Estudios; encargando y fomentando a los estudiantes trabajos de investigación que tengan su estructura propia.

\section{Competencia Profesional:}

El profesional Contador Público debe estar habilitado para.

- Diseñar y aplicar sistemas de información que permitan la toma de decisiones en el corto y largo plazo.

- Usar los costos y conocer las variables que los afecten para la toma de decisiones gerenciales.

- Aplicar las herramientas computacionales para formular simulaciones que le permitan analizar el impacto financiero en diferentes escenarios económicos.

- Analizar la estructura financiera, determinando la estructura óptima y evaluar los riesgos inherentes.

- Establecer políticas para el adecuado manejo financiero de clientes, proveedores, inventarios y el capital de trabajo.

- Evaluar los mecanismos de financiamiento y de inversión que pueda realizar la empresa, evaluando el impacto sobre la estructura financiera de la empresa y su rentabilidad, determinando los riesgos.

- Diseñar sistemas contables para el registro de operaciones financieras y hechos económicos de acuerdo a la teoría contable, usando herramientas computacionales.

- Formular, analizar e interpretar estados financieros básicos en forma individual y consolidada, así como la conversión a moneda extranjera, para la toma de decisiones.

- Conocer las diferencias entre las normas y principios de contabilidad aplicados con nuestro país con el contexto internacional en el registro contable y la formulación de los estados financieros. 
- Aplicar las normas y procedimientos de auditoría para la realización de auditoría financiera, operativa, tributaria y para la formulación del dictamen e informes correspondientes.

- Diseñar, implementar y evaluar sistemas de control interno.

- Aplicar los medios computarizados para la realización de las labores de auditoría.

- Interpretar adecuadamente las disposiciones tributarias que afectan a las personas naturales y jurídicas, realizando su aplicación y planeamiento tributario.

- Conocer las disposiciones tributarias y fiscales de los países con los cuales el Perú tiene convenios de integración, complementación económica o de otra naturaleza.

\section{Campo de Actividad:}

El profesional Contador Público se podrá desempeñar:

- En la actividad pública y privada como Contador General, Auditor Interno, Asesor Tributario, Consultor de Empresas.

- En la docencia universitaria.

- Como perito contable judicial.

- En la función de Auditor Independiente.

- Como árbitro independiente.

Al término de la lectura de este Perfil de Contador público, queda como importante corolario la necesidad de su actualización en función de los cambios que hoy en día tiene la profesión de Contador Público en el mundo; y de los requerimientos mínimos que actualmente se piden a los egresados de las universidades peruanas por parte de empresas privadas e instituciones públicas, empresarios de las pequeñas empresas, bancos y financieras; tomando en cuenta las recomendaciones que salen de im seminarios sobre temas de relación $\dot{x}$ Universidad, Empresa y Estado que ausoıca la Asamblea Nacional de Rectores, parz ofrecer un Contador Público capacitado e el ejercicio de su profesión, tanto al Per_ como a nivel regional en el contexto de Comunidad Andina y de otros pactos regionales en América y el mundo.

Sobre ese futuro Perfil del Contadcr Público que requiere la Facultad de Ciencias Contables de la UNMSM, es importante tomar en cuenta lo que dice el Dr. Antonic C. Nasi. En primer lugar plantea:

\section{ALGUNAS REFLEXIONES SOBRE EL FUTURO DEL CONTADOR PÚBLICO:}

\section{TRANSFORMACIONES QUE IMPACTAN EN EL EJERCICIO PROFESIONAL}

-Examen de Suficiencia

- Examen de Competencia Profesional

- Programas de Educación Continuada Obligatorios

- Control de Calidad Obligatorio

- Nuevo Perfil para un Contador Público Internacional

\section{FORMACIÓN DEL CONTADOR PÚBLICO}

- La Universidad debe conocer lo que es la profesión y formar profesionales y ciudadanos

- Debe exigir al alumno una práctica profesional obligatoria durante su formación universitaria

- Los currículos deben estar actualizados $\mathrm{y}$ coherentes con las necesidades profesionales 
- La enseñanza a distancia será una nueva realidad

- La integración de universidades para los estudios de Postgrado

- La universidad virtual

- La vinculación del profesional con su universidad

- La acreditación universitaria

\section{EXAMEN DE SUFICIENCIA/} COMPETENCIA PROFESIONAL

- Examen para el ingreso a la profesión

- Examen para demostrar la actualización profesional

\section{INTEGRACIÓN CON LA SOCIEDAD}

- Acción de los Organismos Profesionales para promover la profesión ante la sociedad

- Aclaración permanente ante usuarios y la sociedad, del papel del Contador Público, en especial al Auditor Independiente por sus responsabilidades sociales.

A continuación el Dr. Nasi propone el siguiente Perfil de Contador Público en el marco de los próximos cinco años, tomando en cuenta la necesidad de actualización permanente:

PERFIL DE CONTADOR PÚBLICO PARA LOS PRÓXIMOS CINCO AÑOS

- Tener una visión global de la economía y del entorno macroeconómico de los usuarios

- Formar alianzas profesionales para dar a los usuarios una cobertura total
- Conocer fundamentalmente las NICs, NIAs, Normas Nacionales, Normas Éticas, etcétera.

- Conocer los mercados de capitales y financieras globales

- Tener conocimientos humanísticos

- Capacidad de comunicarse

- Hablar más de un idioma (inglés)

- Capacidad para atender usuarios de otros países

- Conocer técnicas de gestión empresarial, en especial en tecnología de información

- Conocimientos de informática, matemáticas estadística y marketing

- Dominio de la Internet, en especial en asuntos de negocios

- Conocimiento de legislación tributaria y societaria de otros países

- Conocimiento de negocios internacionales y regulaciones de entidades del campo

- El Contador Público como gestor del Conocimiento de la Empresa

La segunda parte del presente artículo para la revista Quipukamayoc de la Facultad de Ciencias Contables de la UNMSM, es mostrar los perfiles del Magíster en Contabilidad y del Doctor en Ciencias Contables y Empresariales, cuya responsabilidad de formación la tiene la Unidad de Postgrado, que inició su funcionamiento el 22 de febrero de 1990, es decir ya tiene más de once años de experiencia en el campo de los estudios de postgrado, acogiendo a Contadores Públicos, Licenciados en Administración, Economistas, Abogados, Ingenieros de diversas especialidades y con una gran apertura a otras profesiones que tengan vinculación con áreas de las ciencias de la economía.

Actualmente, funciona el Programa de Maestría en Contabilidad con cinco menciones. Sus perfiles de Maestría en Contabilidad son los siguientes: 


\section{PERFIL DE LA MENCIÓN AUDITORÍA SUPERIOR}

Al terminar sus estudios, el egresado de Maestría en Contabilidad con Mención en Auditoría Superior, será capaz de:

- Desempeñar cargos gerenciales en una sociedad de auditoría así como de auditor independiente.

- Ejercer liderazgo en la formación y desarrollo de una sociedad de auditoría.

- Formular, ejercer y difundir investigaciones en los campos de la producción de la auditoría integral y en la docencia universitaria.

- Proponer modelos innovadores en relación a los procedimientos $\mathrm{y}$ alcances de las diversas disciplinas y modalidades de auditoría existente.

- Ejercer docencia universitaria a nivel de pre y postgrado aplicando modelos pedagógicos innovados.

\section{PERFIL DE LA MENCIÓN BANCA Y FINANZAS}

Al terminar sus estudios, el egresado de Maestría en Contabilidad con Mención en Banca y Finanzas, será capaz de:

- Asesorar a empresas en las áreas de finanzas y gestión de carteras y valores.

- Ejercer la consultoría financiera y bancaria a empresas privadas e instituciones estatales.

- Formular, ejercer y difundir investigaciones en los campos de las finanzas y la docencia universitaria.

- Ejercer docencia universitaria de pre y postgrado utilizando metodologías innovadas.

\section{PERFIL DE LA MENCIÓN CONTABILIDAD DE GESTIÓN}

Al terminar sus estudios, el egresado de Maestría en Contabilidad con Mención en Contabilidad de Gestión, será capaz de:

- Ejercer funciones ejecutivas de: contador gerencial, gerente administrativo y director de empresas.

- Realizar asesoría y/o consultoría a empresas públicas y privadas.

- Formular, ejercer y difundir investigaciones en los campos de la contabilidad, gestión empresarial y en la docencia universitaria.

- Ejercer docencia universitaria a nivel de pre y postgrado usando metodologías innovadas.

\section{PERFIL DE LA MENCIÓN COSTOS Y PRESUPUESTOS}

Al terminar sus estudios, el egresado de Maestría en Contabilidad con Mención en Costos y Presupuestos, será capaz de:

- Ejercer consultoría y asesoría de alto nivel en el diseño, implementación y desarrollo de los costos de producción, comercialización y de servicios.

- Asesorar sectores de explotación de recursos naturales en la solución de problemas macroeconómicos y microeconómicos.

- Ejercer liderazgo en el desarrollo industrial del país a nivel microeconómico.

- Formular, ejercer y difundir investigaciones en los campos de la producción de bienes y servicios, así como de la docencia universitaria.

- Ejercer docencia universitaria de pre y postgrado aplicando modelos pedagógicos innovadas. 


\section{PERFIL DE LA MENCIÓN POLÍTICA Y ADMINISTRACIÓN TRIBUTARIA}

Al terminar sus estudios, el egresado de Maestría en Contabilidad con Mención en Política y Administración Tributaria, será capazde:

- Proporcionar asesoría y/o consultoría sobre políticas tributarias empresariales a nivel de gobierno central, regional y local.

- Formular, ejercer y difundir investigaciones en los campos de la tributación y en la docencia universitaria.

- Ejercer docencia universitaria a nivel de pre y postgrado usando metodologías innovadas.

\section{PERFIL DE DOCTOR EN CIENCIAS CONTABLES Y EMPRESARIALES}

Al término de los estudios, el participante debe alcanzar el perfil de Doctor en Ciencias Contables y Empresariales, cuyas características son las siguientes:

\section{CARACTERÍSTICAS PERSONALES}

a) Poseer un dominio completo de las Ciencias Contables, y una alta especialización en la línea de investigación contable que cultiva.

b) Poseer cultura filosófica y científica lo suficiente formada para ser capaz de comprender la problemática de la ciencia contable en relación al contexto económico, político y social.

c) Ser un investigador reflexivo y crítico de sus propios trabajos.

d) Asumir una posición filosófica para fundamentar sus trabajos. e) Ser un ideólogo como agente de cambio social.

f) Poseer don de gente y buenos modales.

\section{CARACTERÍSTICAS FUNCIONALES}

El doctor en Ciencias Contables y Empresariales, puede desempeñar las siguientes funciones:

a) Investigador académico en Ciencias Contables y Empresariales, en diversas líneas de investigación.

b) Investigador científico y tecnológico en desarrollo de la Gestión Empresarial.

c) Docente Universitario a nivel de Postgrado.

d) Consultor y asesor de alto nivel.

También los Perfiles de Magister en Contabilidad y de Doctor en Ciencias Contables y Empresariales aquí presentados, necesitan renovación para que se mantengan al nivel nacional que se ha alcanzado en más de once años. Los Planes de Estudios de la Unidad de Postgrado, por ende, requieren actualización curricular, agregando las innovaciones tecnológicas, científicas y profesionales que ya se conocen en estos albores del Siglo XXI.

Las puertas de la Unidad de Postgrado, están abiertas a todo Contador Público, sin diferenciar su universidad de procedencia; de igual forma para los demás profesionales que deseen obtener estos Grados Académicos.

¡Honor a la profesión del Contador Público que forma la Facultad de Ciencias Contables de la UNMSM!

¡Honor a los Contadores Públicos y profesionales de otras ramas, que son formados en los estudios de la Unidad de 
Postgrado, en Maestría en Contabilidad y Doctorado en Ciencias Contables, para el desarrollo profesional, en la docencia universitaria y en la investigación científica, que tanto necesita el Perú! 